\title{
Microwave Methods - Evidence to Support a Microwave Effect
}

Mark A. Sanders*, Tracy E. Anderson* and Richard Giberson**

*Imaging Center, College of Biological Sciences, University of Minnesota, 1475 Gortner Ave., St. Paul, MN 55108; **Ted Pella, Inc., Redding, CA 96049

The use of microwave radiation to promote chemical synthesis, tissue fixation and processing for microscopic examination and immunolabeling is becoming accepted methodology. Recent literature pertaining to chemical synthesis of nanoparticles [1] and EDTA decalcification of osseous tissue [2] reinforce that a microwave effect exists. Improved microwave technology has led to improved control over the microwave environment during processing [3]. These advancements have made it possible to examine the role of microwave radiation independent of microwave heating [2]. This submission presents further evidence in support of a microwave effect with respect to the formaldehyde fixation process and diffusion dynamics at the cellular level.

Formaldehyde used either as 10\% neutral buffered formalin or buffered $4 \%$ paraformaldehyde has been described as quick to penetrate and slow to fix; in part due to the chemical equilibrium that results when the fixative is mixed with water [4]. In the past when fresh tissue was placed in the fixative and exposed to microwave radiation the process met with failure unless the tissue was infiltrated with fixative prior to microwave exposure. Current work has demonstrated that microwave-assisted formaldehyde fixation is a two-step (low-150W followed by high-650W) wattage dependent process which is independent of microwave induced sample heating. Samples under identical conditions but without microwave radiation failed to show a similar degree of sample preservation as that seen from microwave exposed tissue when compared by transmission electron microscopy, H\&E stained paraffin sections and immunohistochemistry.

In addition to tissue fixation, very low wattage in a dedicated laboratory microwave ( $<250$ watts) can also be used to facilitate access of probes into tissue culture cells. The present study will have two objectives: to rapidly prepare specimens for fluorescent imaging utilizing low wattage, temperature controlled microwave energy in in vivo experiments in animal tissue culture cells. Low power microwave energy was used in conjunction with a modified microwave accessory called the ColdSpot ${ }^{\mathrm{TM}}$ (Ted Pella, Inc., Redding, CA) that creates a uniform environment of microwave irradiation to the samples.

We have previously shown that under reduced wattage conditions ( $\sim 150$ watts), living plant and animal tissue can be made more "permeable" to fluorescent vital dyes such as the Syto series of nucleic acid stains (Molecular Probes, Eugene OR). Brief microwave treatment in the presence of the stain greatly increased the depth and rate of penetration of the dye.

This report demonstrates the use of low power microwave energy (150-250W) in conjunction with a modified ColdSpot ${ }^{\mathrm{TM}}$ for the uptake of cell permeable and impermeable fluorescent probes. HeLa tissue culture cells were simultaneously or sequentially exposed to the cell permeable nucleic acid dye Syto 13 and the cell impermeable fluorescent probes propidium iodide or fluorescenated dextran $(1200 \mathrm{kD})$ in the presence or absence of microwave energy. The reported results indicate low power microwave energy combined with better control of the microwave environment induced the uptake of cell impermient probes within minutes. The viability assay confirmed that $>85 \%$ of the cells were still viable following 4 minutes of microwave treatment.

The results indicate that the typically cell impermeant probes gain access to interior compartments of the cell in the presence of microwaves and that these microwave treatments maintain a high degree of cellular viability.

In conclusion, this method increases the access of reagents used for labeling and the 
preservation. The reported procedures can have broad reaching applications in the preparation of specimens in clinical and research laboratory environments.

References

[1]Gerbec JA, Magana D, Washington A, Strouse GF. Microwave-enhanced reaction rates for nanoparticle synthesis. J Am Chem Soc. 2005 Nov 16;127(45):15791-800.

[2]Tinling, S.P. Giberson, R.T., Kullar, R.S. (2004) Microwave exposure increases bone demineralization rate independent of temperature. J. Microsc., 215:230-235.

[3]Galvez, J.J., Giberson, R.T., Cardiff, R.D. (2004) Microwave mechanisms - the energy/heat dichotomy. Microsc. Today, 12(2):18-23.

[4]Fox CH, Johnson FB, Whiting J, Roller PP (1983) Formaldehyde fixation. J Histochem Cytochem $33: 845-853$.
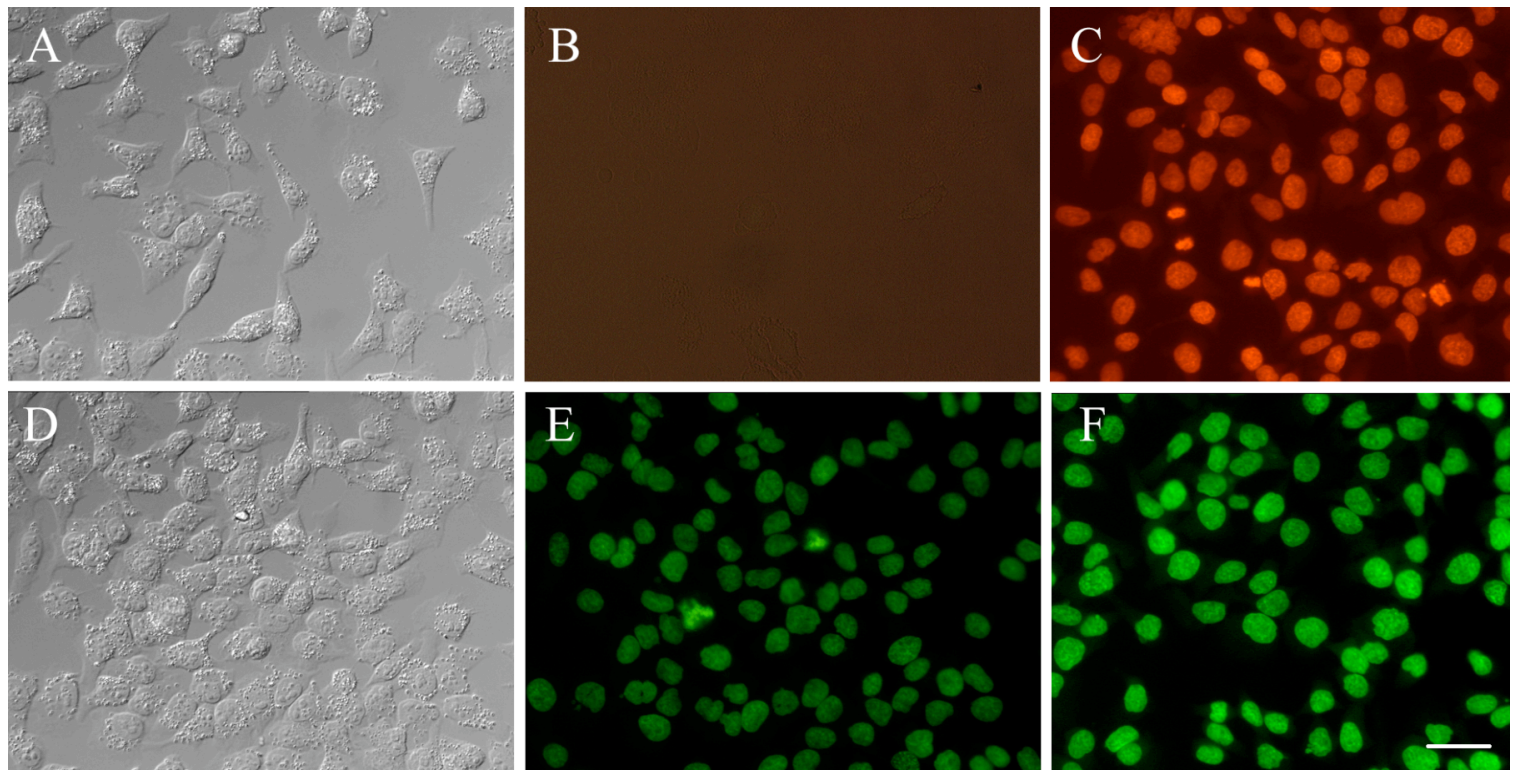

Figure 1. A-C. Hela cells treated with $1 \mu \mathrm{M}$ propidium iodide. A. DIC. B. Fluorescent image following labeling for 5 minutes at $37^{\circ} \mathrm{C}$. C. Fluorescent image following 4 minutes exposure to $150 \mathrm{~W}$ microwave irradiation ( 1.75 minutes on, 0.5 minutes off, 1.75 minutes on) at $37^{\circ} \mathrm{C}$. D-F. Hela cells treated with $1 \mu \mathrm{M}$ Syto 16. D. DIC. E. Fluorescent image following labeling for 5 minutes at $37^{\circ} \mathrm{C}$. F. Fluorescent image following 4 minutes exposure to $150 \mathrm{~W}$ microwave irradiation $(1.75$ minutes on, 0.5 minutes off, 1.75 minutes on) at $37^{\circ} \mathrm{C}$. Scale bar $=10$ microns.
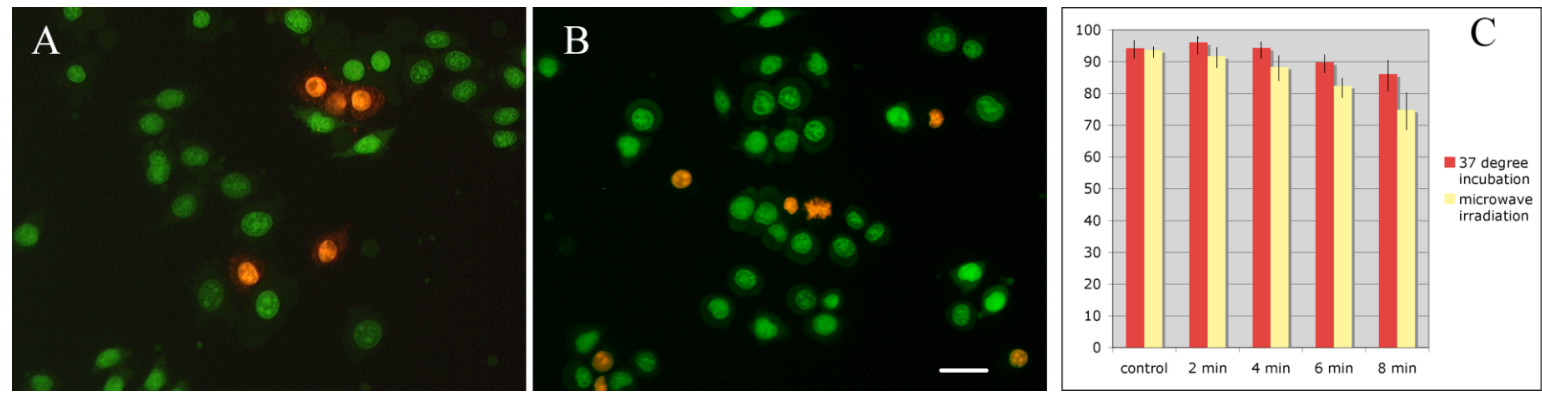

Figure 2. A-B HeLa cells labeled with Live (green)/Dead(red) probe for 6 minutes in the absence (A) and presence (B) of $150 \mathrm{~W}$ microwave irradiation (2.5 minutes on, 1 minute off, 2.5 minutes on). C. Graph of percent of viable cells following MWI at a microwave power setting of approximately 150 watts (\# 1 power level). Approximately 300 cells for each treatment were used in the analysis. Scale bar $=10$ microns. 